\title{
Probing the dye-semiconductor interface in dye-sensitized NiO solar cells
}

Nathan T. Z. Potts ${ }^{a}$, Tamara Sloboda ${ }^{d}$, Maria Wächtler, ${ }^{b, c}$ Ruri Agung Wahyuono ${ }^{b, c}$, Valeria D'Annibale $^{a}$, Benjamin Dietzek, ${ }^{\mathrm{b}, \mathrm{c}}$ Ute B. Cappel ${ }^{\mathrm{d}}$, Elizabeth A. Gibson ${ }^{\mathrm{a} *}$

a Energy Materials Laboratory, Chemistry: School of Natural and Environmental Science, Newcastle University, Newcastle upon Tyne, NE1 7RU, UK. Elizabeth.gibson@ncl.ac.uk, ORCID 0000-0002-6032-343X

b Leibniz-Institute of Photonic Technology (IPHT) Jena e.V., Department Functional Interfaces, Albert-Einstein-Straße 9, 07745 Jena, Germany

${ }^{c}$ Institute of Physical Chemistry and Center for Energy and Environmental Chemistry Jena (CEEC Jena), Friedrich-Schiller-University Jena, Helmholtzweg 4, 07743 Jena, Germany

d Division of Applied Physical Chemistry, Department of Chemistry, KTH Royal Institute of Technology, SE-100 44 Stockholm, Sweden.

\begin{abstract}
The development of $p$-type dye sensitized solar cells (p-DSSCs) offers an opportunity to assemble tandem photoelectrochemical solar cells with higher efficiencies than $\mathrm{TiO}_{2}$-based photoanodes, pioneered by Grätzel and O'Regan. This paper describes an investigation into the behavior at the interfaces in p-DSSCs, using a series of bodipy dyes, BOD1-3. The three dyes have different structural and electronic properties which lead to different performance in p-DSSCs. We have applied photoelectron spectroscopy and transient absorption spectroscopy to rationalize these differences. The results show that the electronic orbitals of the dyes are appropriately aligned with the valence band of the $\mathrm{NiO}$ semiconductor to promote light-induced charge-transfer, but charge-recombination is too fast for efficient dye-
\end{abstract}


regeneration by the electrolyte. We attribute this fast recombination, which limits the efficiency of the solar cells, to the electronic structure of the dye and the presence of $\mathrm{Ni}^{3+}$ recombination sites at the NiO surface.

\section{Introduction}

The aim of this study was to probe the energy alignment and electron transfer dynamics at the interface between a series of three bodipy dyes and NiO. This light induced charge transfer at this interface is key to the efficiency of dye-sensitized photocathodes in p-type and tandem dye sensitized solar cells (DSSCs). While the dye|semiconductor interface is well understood with n-type metal oxides such as $\mathrm{TiO}_{2}$, much less is known for $\mathrm{p}$-type semiconductors such as $\mathrm{NiO}$. The information learned is useful to understand why NiO DSCs perform less well than $\mathrm{TiO}_{2}$ DSSCs and to develop new approaches to improving p-type DSSCs.

Dye sensitized solar cells are photoelectrochemical devices which contain a thin film of a transparent, mesoscopic semiconductor, such as $\mathrm{TiO}_{2}$, coated with dye molecules to absorb light. ${ }^{1}$ Light induced charge-separation takes place at this interface, followed by diffusion of electrons through the $\mathrm{TiO}_{2}$ to the conductive substrate (typically conductive glass), and diffusion of an oxidized species in an electrolyte solution to "regenerate" the ground state of the dye. The reduced electrolyte species is re-oxidized at a counter electrode, typically platinized conductive glass, to complete the circuit. ${ }^{1}$ Low cost processing methods, such as screen or ink-jet printing, can be used to deposit the materials, so the technology lends itself to roll to roll production and a range of substrates such as glass, plastic and metal foils can be used. $^{2}$ The devices are semi-transparent and the choice of colors provides an opportunity for artistic designs and building-integrated photovoltaics. ${ }^{3,4}$ A key benefit of the DSSC over established photovoltaic technology is the relatively high efficiency of DSSC under low or diffuse light conditions (i.e. indoors or overcast days). Technologies with up to $28.9 \%$ efficiency 
under these low light conditions have been achieved, opening possibilities for powering small electronic devices within the home. ${ }^{5}$ However, under standard testing conditions (AM1.5, 1 $\mathrm{kW} \mathrm{cm} \mathrm{cm}^{-2}$ irradiance) the highest efficiency reached for n-type DSSCs is $14.3 \%$, and only incremental improvements to performance have been made over the last 20 years. ${ }^{6}$

Pairing an n-type $\left(\mathrm{TiO}_{2}\right)$ photoanode and a p-type photocathode in a tandem DSSC increases the theoretical maximum efficiency by adding the voltage of the individual devices. Using complimentary dyes, e.g. harvesting high energy photons on the n-type photoanode and lower energy photons on the p-type photocathode, would harness a larger portion of the solar spectrum by reducing the spectral overlap of the dyes. ${ }^{7}$ Much research has been invested in $\mathrm{TiO}_{2}$-based devices, but p-type dye-sensitized solar cells are less developed and the efficiencies are considerably lower. ${ }^{8}$ Typically, $\mathrm{NiO}$ is chosen as the transparent, mesoporous p-type semiconductor. The mechanism differs as electrons are transferred from the valence band of the $\mathrm{NiO}$ to the photoexcited dye and the holes which are produces then diffuse through the $\mathrm{NiO}$ electrode to the conductive glass substrate, while the reduced dye is regenerated by the electrolyte. The standard redox electrolyte contains iodide/triiodide for compatibility with typical $\mathrm{TiO}_{2}$-based DSSCs. As with n-type DSSCs, there are several recombination processes that lead to losses in the device. These are shown in Figure 1, together with the typical lifetimes for the processes. For a high-efficiency device, the lifetimes for the forward processes (driving the photocurrent) must be much longer than the reverse (charge-recombination) processes. Therefore, studying the electronic properties and electron-transfer dynamics at the dyesemiconductor interface in p-type DSSCs is essential to developing better materials and higher performing devices. 


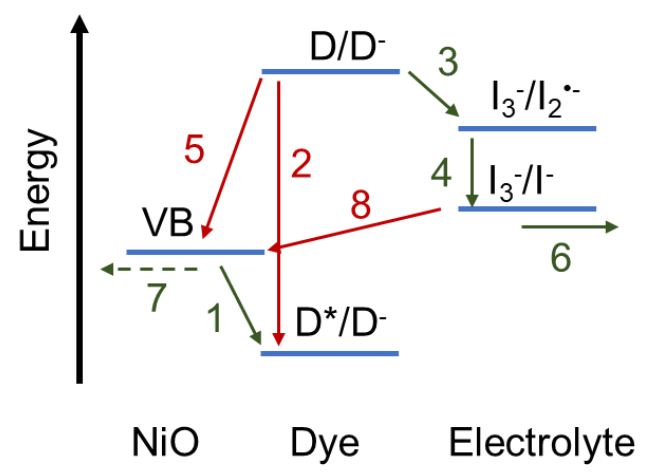

1. Charge injection: Electron transfer from the NiO valence band to the excited dye. $\mathrm{T}<10 \mathrm{ps}$

2. Radiative or non-radiative relaxation of the dye excited state. $\mathrm{T} \approx \mathrm{ns}-\mu \mathrm{s}$

3. Regeneration: Electron transfer from the reduced dye to the oxidised species in the electrolyte. $\mathrm{T} \approx 20 \mathrm{~ns}-10 \mu \mathrm{s}$

4. Disproportionation of $\mathrm{I}_{2} \odot$. $\mathrm{T} \approx 0.3 \mathrm{~ns}$

5. Charge recombination between the dye radical anion and a hole in the $\mathrm{NiO}$. $\mathrm{T} \approx 10 \mathrm{ps}-30 \mu \mathrm{s}$

6. Diffusion of the reduced species in the electrolyte to the counter electrode for re-oxidation.

7. Diffusion of holes in the $\mathrm{NiO}$ to the conductive glass substrate. $\mathrm{T} \approx 50-100 \mathrm{~ms}$

8. Charge recombination between the reduced species in the electrolyte and a hole in the NiO. $\mathrm{T}>0.1 \mathrm{~s}$

Figure 1. Charge transfer processes in p-type DSSCs following photoexcitation.

Much of the research into p-type DSSCs has focused on developing new photosensitizers. The requirements are as follows. High absorption coefficients are necessary due to the limited film thickness of the $\mathrm{NiO}$ electrode (c.a $1.5-3 \mu \mathrm{m}){ }^{.9}$ An anchoring group, typically a carboxylic acid, is necessary to facilitate strong adsorption onto the metal oxide surface and to enable efficient photoinduced charge transfer at the interface. ${ }^{10}$ The HOMO of the dye must be positioned at a more positive potential than the valence band edge of the semiconductor and the LUMO must be more negative than the redox potential of the electrolyte. ${ }^{11}$ The dye should be stable under operational conditions and the absorption spectra should span the region transmitted by $\mathrm{TiO}_{2}$ photoanodes, which is typically the red-NIR. ${ }^{12,13}$ Bodipy dyes are ideal for tandem cells because they have high absorption coefficients, their reduced forms are stable 
and the optical and electrochemical properties can easily be tuned with simple chemical transformations.

We have previously shown how the electronic communication with the $\mathrm{NiO}$ can be optimized by structural changes, such as tuning the angle between the chromophore and anchoring group and adding electron donating/accepting groups. ${ }^{14}$ In this paper, we used simpler dye structures with intense, characteristic spectroscopic signals to examine the interfaces of the dye with the NiO surface and the electrolyte. This approach should enable us to distinguish between different intermediates and electronic states, despite low concentrations. A series of three bodipy dyes, BOD1-3 (Figure 2), each with a carboxylic acid anchoring group, were prepared and characterized. We then examined how the structural and electronic properties of the dyes affected the p-DSSC characteristics and then performed a series of experiments to examine the electronic properties and electron-transfer dynamics in the photocathodes. BOD1 was previously shown to be an effective photosensitizer for NiO DSSCs. ${ }^{14}$ The methyl substituents at the 1,7 positions fix the benzoic acid anchoring group perpendicular from the dipyrrene, so the chromophore is electronically decoupled from the NiO surface. The redox potentials and electrochemical stability of BOD1 are appropriate for NiO. However, the absorption spectrum $\left(\lambda_{\max }=528 \mathrm{~nm}\right.$ in $\left.\mathrm{CH}_{2} \mathrm{Cl}_{2}\right)$ is unfavorable for tandem devices as it overlaps with the region of the visible spectrum where state of the art dyes for $n$-type DSSCs operate. In this study, we prepared two derivatives, BOD2 and BOD3, in which the methyl substituents are replaced by an aromatic subsituent, enabling rotational freedom around the benzoic aciddipyrrene axis. These new dyes have broader absorption and emission bands, which are shifted bathochromically compared to BOD1. This enables more light harvesting across the optical spectrum, which could be exploited either in a tandem device or a co-sensitized cell. BOD3 contains phenol (rather than the phenyl substituents in BOD2) which extends the chromophore through B-O coordination. ${ }^{15}$ This approach, forming a chiral, boron-chelated bodipy, has been used previously for applications requiring red/NIR-absorbing/emitting 
dyes. ${ }^{16-19}$ This manuscript describes the impact of these structural modifications on the properties of $\mathrm{NiO}$ photocathodes.

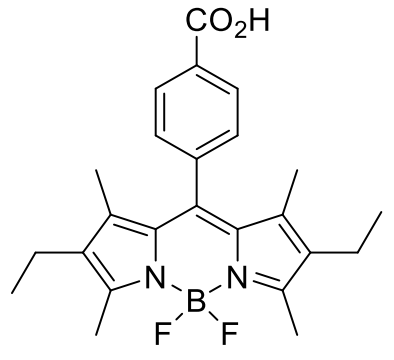

BOD1

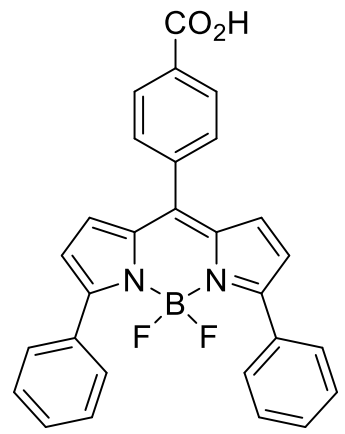

BOD2

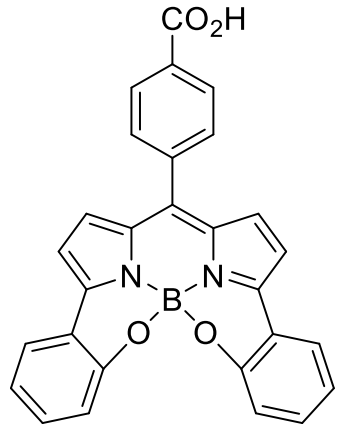

BOD3

Figure 2. Structures of the series of dyes, BOD1-3, investigated in this paper.

\section{Results}

\section{Synthesis and characterisation of the photosensitizers}

The dyes BOD1-3 were synthesised as described in the supporting information. Initial attempts using regioselective halogenation of the bodipy core and subsequent deprotection of the methyl ester protecting group were unsuccessful, a reworked synthesis pathway provided two novel bodipy dyes with benzoic acid anchor groups suitable for use in a DSSC. 


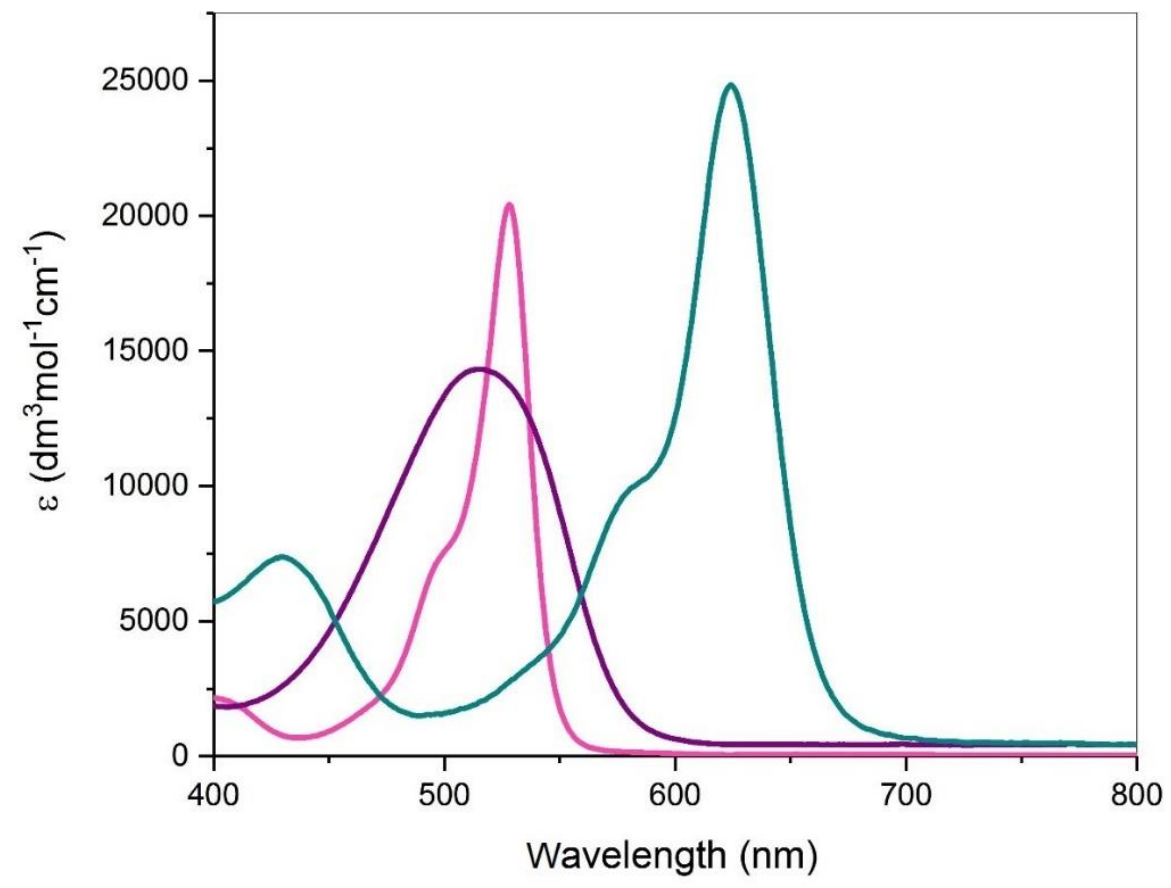

Figure 3. UV-Visible absorption spectra of BOD1 (pink), BOD2 (purple) and BOD3 (green) recorded in dry, degassed acetonitrile.

Table I: Photophysical properties of BOD1, BOD2 and BOD3 in acetonitrile solution.

\begin{tabular}{|l|l|l|l|}
\hline & $\begin{array}{l}\lambda_{\text {abs }} / \mathbf{n m} \\
\left(\varepsilon / \mathbf{d m}^{3} \mathbf{m o l}^{-1} \mathbf{c m}^{-1}\right)\end{array}$ & $\lambda_{\text {em }} / \mathbf{n m}$ & $E_{0-0} / \mathbf{e V}$ \\
\hline BOD1 & $528(20400)$ & 536 & 2.33 \\
\hline BOD2 & $515(14300)$ & $541^{*}$ & $2.35^{\star}$ \\
\hline BOD3 & $625(24800)$ & 654 & 1.93 \\
\hline
\end{tabular}

* $538 \mathrm{~nm}$ excitation 
The photophysical properties of the dyes BOD1-3 are provided in Table I, the absorption spectra are given in Figure 3 and the emission spectra are given is Figure S2. BOD1 exhibits absorption and emission spectra that are characteristic of a bodipy So to S1 transition. BOD2 has a much broader absorption spectrum then BOD1. A hypsochromic shift of ca. $13 \mathrm{~nm}$ and a lower molar absorption coefficient was observed when the $\alpha, \beta$ alkyl groups of BOD1 were substituted for a-phenyl substituents in BOD2. The emission spectra of BOD2 similarly contain weak, broad bands with different intensities. The weak fluorescence is consistent with similar bodipy systems with phenyl substituents which are able to rotate freely. ${ }^{20}$ The excitation spectra (Figure S3) associated with these two peaks reveal the presence of two emissive species in solution. Emission at $538 \mathrm{~nm}$ is associated with an excitation spectrum containing a narrow band with a shoulder at higher energy than the maximum, which is typical for bodipy. Excitation at $580 \mathrm{~nm}$ is associated with a broad featureless excitation spectrum, lower in intensity and bathochromically shifted by $8 \mathrm{~nm}$ compared to the excitation spectra associated with the higher energy emission band. This is consistent with the formation of $\mathrm{J}$-aggregates. ${ }^{21}$ In BOD3, B-O coordination forces the phenyl groups towards the plane of the dipyrromethene chromophore. Accordingly, both the absorption and emission of BOD3 are bathochromically shifted compared to both BOD1 and BOD2, and the bands are more intense.

The redox potentials of BOD1-3 were measured in acetonitrile solution to determine their suitability for use in a p-type DSSC. We were unable to reduce the dye when directly attached to the semiconductor because the reduction potential lies in the $\mathrm{NiO}$ band gap. The results from cyclic voltammetry and differential pulse voltammetry in acetonitrile solution are provided in the supporting information and are summarized in Table II. The reduction (BOD/BOD') was reversible for all three dyes, but the oxidation (BOD $/ B O D)$ was only reversible for BOD1. The redox potentials were used to estimate the driving force for charge transfer between the dye and the semiconductor, which are included in Table II. $\Delta G_{\text {inj }}$ was substantial for all three dyes, highest for BOD2 and lowest for BOD3. $\triangle G_{\text {reg }}$ also was favorable for all three dyes but was highest for the alkyl substituted BOD1. 
Table II. Electrochemical properties of BOD1-3 in acetonitrile solution with $0.5 \mathrm{M} \mathrm{TBACIO}$ and calculated driving forces for electron transfer processes within p-DSSC device. All potentials are reported vs $\mathrm{Fc} / \mathrm{Fc}^{+}$.

\begin{tabular}{|c|c|c|c|c|c|c|}
\hline & $E\left(D^{+} / D\right) / V$ & $E\left(D^{\prime} / D^{-}\right) / V$ & $E\left(D^{*} / D^{-}\right) / V$ & $\Delta G_{\text {inj }} / \mathrm{V}$ & $\Delta G_{\text {reg }} / \mathrm{V}$ & $\Delta G_{\text {rec }} / \mathrm{V}$ \\
\hline BOD1 & 0.62 & -1.54 & 0.79 & -0.91 & -0.72 & -1.42 \\
\hline BOD2 & 0.67 & $\begin{array}{l}-1.28 \\
\end{array}$ & 1.07 & -1.19 & -0.46 & -1.16 \\
\hline BOD3 & 0.69 & -1.29 & 0.64 & -0.76 & -0.47 & -1.17 \\
\hline
\end{tabular}

$E\left(\mathrm{D}^{+} / \mathrm{D}\right)$ (ground state oxidation potential) and $E\left(\mathrm{D} / \mathrm{D}^{-}\right)$(ground state reduction potential) were determined from the differential pulse voltammograms. $E\left(D^{*} / D^{-}\right)$(excited state reduction potential) was calculated using $E\left(\mathrm{D}^{*} / \mathrm{D}^{-}\right)=E\left(\mathrm{D}^{-} \mathrm{D}^{-}\right)+E_{0-0 .} \Delta G_{\mathrm{inj}}=\mathrm{e}\left[E_{\mathrm{VB}}(\mathrm{NiO})-E\left(\mathrm{D}^{*} / \mathrm{D}^{-}\right)\right]$and $\Delta G_{\text {reg }}=\mathrm{e}\left[E\left(\mathrm{D} / \mathrm{D}^{-}\right)-E\left(\mathrm{I}_{3}^{-} / \mathrm{l}_{2}^{-}\right)\right], \Delta G_{\text {rec }}=\mathrm{e}\left[E\left(\mathrm{D}^{-} \mathrm{D}^{-}\right)-E_{\mathrm{VB}}(\mathrm{NiO})\right]$ where $\left(E_{\mathrm{VB}}(\mathrm{NiO})=-0.12 \mathrm{~V} \mathrm{vs}\right.$ $\left.\mathrm{Fc} / \mathrm{Fc}^{+}\right), E\left(\mathrm{I}_{3}{ }^{-} / \mathrm{I}^{-}\right)=-0.28 \mathrm{~V}$ vs $\mathrm{Fc} / \mathrm{Fc}^{+}, E\left(\mathrm{I}_{3} / \mathrm{I}_{2}^{-}\right)=-0.82 \mathrm{~V}$ vs $\mathrm{Fc} / \mathrm{Fc}^{+} .22$

\section{Density Functional Theory Calculations}

Density Functional Theory (DFT) calculations were performed to determine the molecular orbital distributions within BOD1-3, to visualize the electronic transitions that occur when the dye is excited. A range of exchange functionals and basis sets were tested and the B3LYP exchange functional and $6-31 G^{*}$ basis set were found to match closest to the trends in experimental data. The HOMO-LUMO orbital distributions for BOD3 are provide in Figure 4. The HOMO-LUMO orbital distributions for BOD1 and BOD2 with their respective orbital and transition energies are provided in Figures S5 and S6. In all cases, the HOMO is distributed 
across the dipyrromethene and pyrrole substituents, but there is node at the meso position and a lack of electron density on the benzoic acid anchoring group. In contrast, the electron density in the LUMO is distributed across the entire molecule, including the anchoring group.
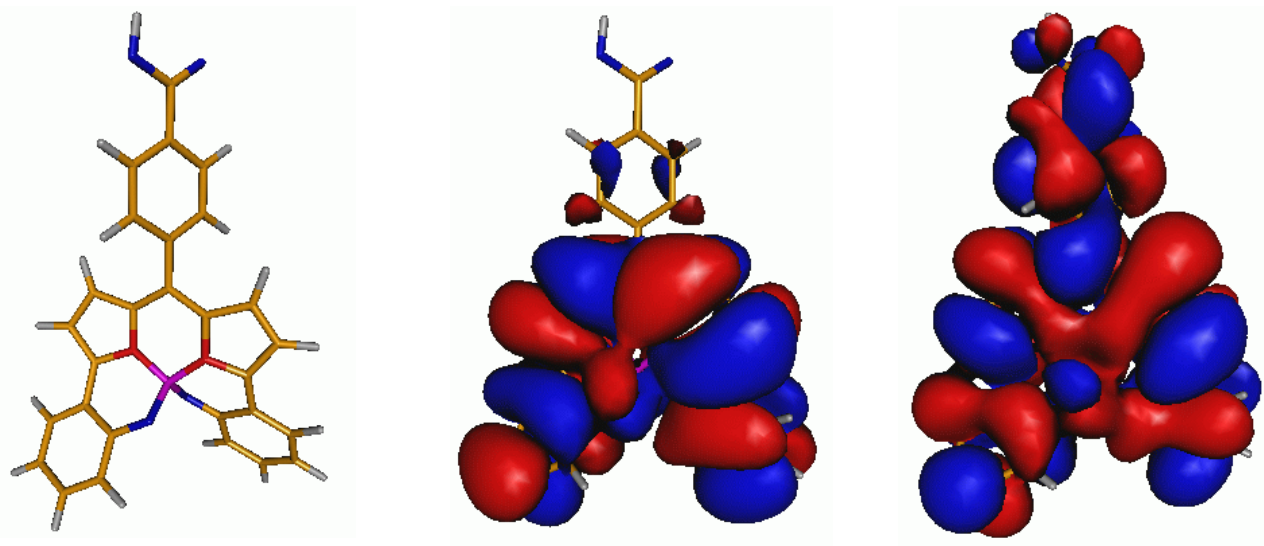

Figure 4. The optimized geometry (left) and the orbital distribution of the HOMO (middle) and LUMO (right) of BOD3 with MeCN solvation calculated using the PCM with B3LYP as functional and $6-31 G^{*}$ basis set.

\section{Dye-sensitized Solar Cell Characteristics}

Nickel oxide dye-sensitized solar cells were assembled with dyes BOD1-3 to compare the photoelectrochemical performance of the three sensitizers. The experimental details are provided in the supporting information. Briefly, mesoporous $\mathrm{NiO}$ thin films on conductive glass were immersed overnight in acetonitrile solutions of the dyes. The UV-visible spectra of the dyes adsorbed on the $\mathrm{NiO}$ surface (Figure S4) broaden compared to the solution spectra, indicating electronic communication between the dye and the $\mathrm{NiO}$. The light harvesting efficiency $(L H E)$ at the maximum wavelength of the dyes has been estimated from these spectra and included in Table III to compare the relative absorbance of the sensitized $\mathrm{NiO}$ films. The photocathodes were combined with a platinum counter electrode using a $25 \mu \mathrm{m}$ thick Surlyn spacer and cells were filled with a redox electrolyte containing iodide/triiodide in acetonitrile. Two triiodide concentrations were used, $0.1 \mathrm{M}$ and $0.5 \mathrm{M}$. Photocurrent density- 
voltage curves were recorded to determine the solar cell characteristics, which are summarized in Table III. For reference, p-DSSCs with P1 as the sensitizer were also tested.

Table III. Photovoltaic performance of nickel oxide dye-sensitized solar cells containing BOD1-3 and reference P1, with an acetonitrile electrolyte prepared by adding 1.0 M Lil and either $0.1 \mathrm{M}$ or $0.5 \mathrm{M} \mathrm{I}_{2}$ to form $\mathrm{I}_{3}$.

\begin{tabular}{|c|c|c|c|c|c|c|}
\hline & {$\left[\mathrm{I}_{3}^{-}\right] / \mathbf{M}$} & $J_{s c} / \mathrm{mA} \mathrm{cm}^{-2}$ & $V_{\text {oc }} / \mathrm{V}$ & $F F / \%$ & $\eta / \%$ & LHE / \% \\
\hline \multirow[t]{2}{*}{ BOD1 } & 0.1 & 0.12 & 0.07 & 36 & 0.004 & \multirow[t]{2}{*}{55} \\
\hline & 0.5 & 0.56 & 0.07 & 38 & 0.015 & \\
\hline \multirow[t]{2}{*}{ BOD2 } & 0.1 & 0.48 & 0.04 & 29 & 0.006 & \multirow[t]{2}{*}{75} \\
\hline & 0.5 & 0.58 & 0.02 & 28 & 0.003 & \\
\hline \multirow[t]{2}{*}{ BOD3 } & 0.1 & 0.21 & 0.06 & 29 & 0.003 & \multirow[t]{2}{*}{65} \\
\hline & 0.5 & 0.42 & 0.02 & 29 & 0.002 & \\
\hline \multirow[t]{2}{*}{ P1 } & 0.1 & 2.12 & 0.12 & 32 & 0.080 & \\
\hline & 0.5 & 2.26 & 0.11 & 33 & 0.080 & \\
\hline
\end{tabular}

$J_{\mathrm{SC}}=$ short circuit current density, $V_{\mathrm{OC}}=$ the open circuit voltage, $F F=$ fill factor, $\eta=$ solar to electrical power conversion efficiency. LHE = Light Harvesting Efficiency at the maximum absorption wavelength $=1-10^{-A}$. The equilibrium constant for triiodide dissociation is $\mathrm{K}=6.3$ $\times 10^{6} \mathrm{~L} \mathrm{~mol}^{-1}$ in acetonitrile. ${ }^{22}$

\section{Hard and Soft X-Ray Photoelectron Spectroscopy}

X-ray photoelectron spectroscopy (PES) has been used previously to characterise the dyesemiconductor interface in n-type $\mathrm{TiO}_{2}$ based devices, however, studies involving p-type $\mathrm{NiO}$ are less common. ${ }^{23,24}$ In this study, the electronic and molecular properties of dyes BOD1BOD3 adsorbed on FTO and NiO were studied with different photon energies. This enabled us to probe the photocathode surfaces at different depths and to investigate the electronic 
states of the dye, the semiconductor and the dye-semiconductor interface. The valence states of the $\mathrm{NiO}$ semiconductor were compared to the $\mathrm{HOMO}$ of the dye to evaluate the driving force for charge-separation in the p-DSSC and to compare this with the values estimated from electrochemistry of the separate components. Core level spectra of the dyes adsorbed on FTO and on NiO are shown in Figures S16 to S19. The binding energy (BE) scale was calibrated using an external gold reference (Au4f $84.00 \mathrm{eV}$ or Fermi level $0.00 \mathrm{eV}$ ).

Table IV. Measured core level binding energies (BE) for dyes BOD1-3 adsorbed on FTO and $\mathrm{NiO}$ at $2200 \mathrm{eV}$ X-ray excitation energy.

\begin{tabular}{|l|l|l|l|}
\hline BOD1 & FTO / eV & NiO / eV & $\mathbf{B E} / \mathbf{e V}$ \\
\hline C1s (main) & 285.3 & 284.9 & -0.4 \\
\hline N1s & 400.1 & 399.3 & -0.8 \\
\hline F1s & 686.2 & 685.8 & -0.4 \\
\hline BOD2 & FTO & NiO & $\mathbf{B E}$ \\
\hline C1s (main) & 285.1 & 284.6 & -0.5 \\
\hline N1s & 399.9 & 399.4 & -0.5 \\
\hline F1s & 686.1 & 685.6 & -0.5 \\
\hline BOD3 & FTO & NiO & $\Delta$ BE \\
\hline C1s (main) & 285.1 & 284.8 & -0.3 \\
\hline N1s & 400.3 & 399.7 & -0.6 \\
\hline
\end{tabular}


Table V. Measured core level binding energies for the substrates FTO and NiO at $2200 \mathrm{eV} \mathrm{X-}$ ray excitation energy in the absence and presence of BOD1-3.

\begin{tabular}{|l|l|l|}
\hline & FTO: Sn3d / eV & NiO: Ni2p (main) / eV \\
\hline FTO only & 487.2 & \\
\hline NiO on FTO & & 853.5 \\
\hline BOD1 & 487.2 & 853.6 \\
\hline BOD2 & & 853.6 \\
\hline BOD3 & 487.2 & 853.6 \\
\hline
\end{tabular}

The C1s, N1s and F1s core level energies for the dye adsorbed on FTO and NiO at $2200 \mathrm{eV}$ photon energy are summarised in Table IV. The core level spectra of the dyes were consistent with the structural differences in BOD1-3. The B1s core level spectra of bodipy dyes can be weak and we were not able to extract an exact binding energy position. However, the synchrotron source enabled us to achieve sufficient signal to noise to observe a shift to lower binding energy for BOD3 compared to BOD1 and BOD2 (Figure S19). This is consistent with the change of coordination, where $\mathrm{B}$ is coordinated to two $\mathrm{N}$ and two $\mathrm{O}$ atoms in BOD3 and to two $\mathrm{N}$ and two $\mathrm{F}$ atoms in BOD1 and BOD2. Likewise, the F1s spectra are consistent with the structural differences, (Figure S17), with signals present at similar binding energies for both BOD1 and BOD2, and absent for BOD3. All three bodipy dyes contain two equivalent nitrogen atoms which appear as one peak in the N1s core level spectra on NiO (Figure S18). BOD3 contains a second nitrogen peak on FTO but not $\mathrm{NiO}$, which seems to be contamination. All core levels for the dyes on $\mathrm{NiO}$ are shifted to lower binding energy and are narrower compared to on FTO (Table IV). The exact magnitude varies slightly for different core levels for BOD1 and BOD3 with the N1s peaks showing a larger shift. For BOD2, the observed shifts are consistently $0.5 \pm 0.05 \mathrm{eV}$. We compared dye loading from the UV-visible 
absorption spectra of the $\mathrm{BOD} \mid \mathrm{NiO}$ and the $\mathrm{BOD} \mid \mathrm{NiO}$ samples, accounting for the differences in absorption coefficient. The amount of dye on FTO was similar for BOD1-3. However, there appeared to be more BOD2 adsorbed on $\mathrm{NiO}$ compared to BOD1|NiO and BOD3|NiO (Figure S4).

Figure S20 and Figure S21 show the valence photoelectron spectra for BOD1-3 deposited on FTO and FTO only at excitation energies of 2200 , and $120 \mathrm{eV}$. As the excitation energy is increased, the shape of the spectra changes as the probing depth increases with the kinetic energy of the measured electrons and as the relative photoionisation cross-sections of the orbitals contributing to the signal can change. A larger contribution from the valence orbitals of the dyes is observed with $120 \mathrm{eV}$, whereas the with $2200 \mathrm{eV}$ the major contribution to these spectra is from the FTO substrate though the $\mathrm{Sn} 4 \mathrm{~d}$ peak at $26.4 \mathrm{eV}$ and its valence band features between 12 and $4 \mathrm{eV}$ binding energy.$^{23}$ Filled states are also observed up to the Fermi level, which have been assigned to bandgap states in the n-doped FTO. ${ }^{25}$ These states are not observed at $120 \mathrm{eV}$, possibly due to relative changes in cross section. Instead, a shoulder or tail before the onset of the FTO VB edge observed with $120 \mathrm{eV}$ excitation energy is assigned to the HOMO levels of the dye (Figure S21). Figure S24 shows the calculation of dye-only spectra obtained through subtracting the FTO signal from the dye on FTO spectra at $120 \mathrm{eV}$ when both spectra had been normalised to the Sn4d intensity. After this analysis, a clear HOMO peak can be observed for BOD2. Broader features were observed for BOD1 and BOD3. 

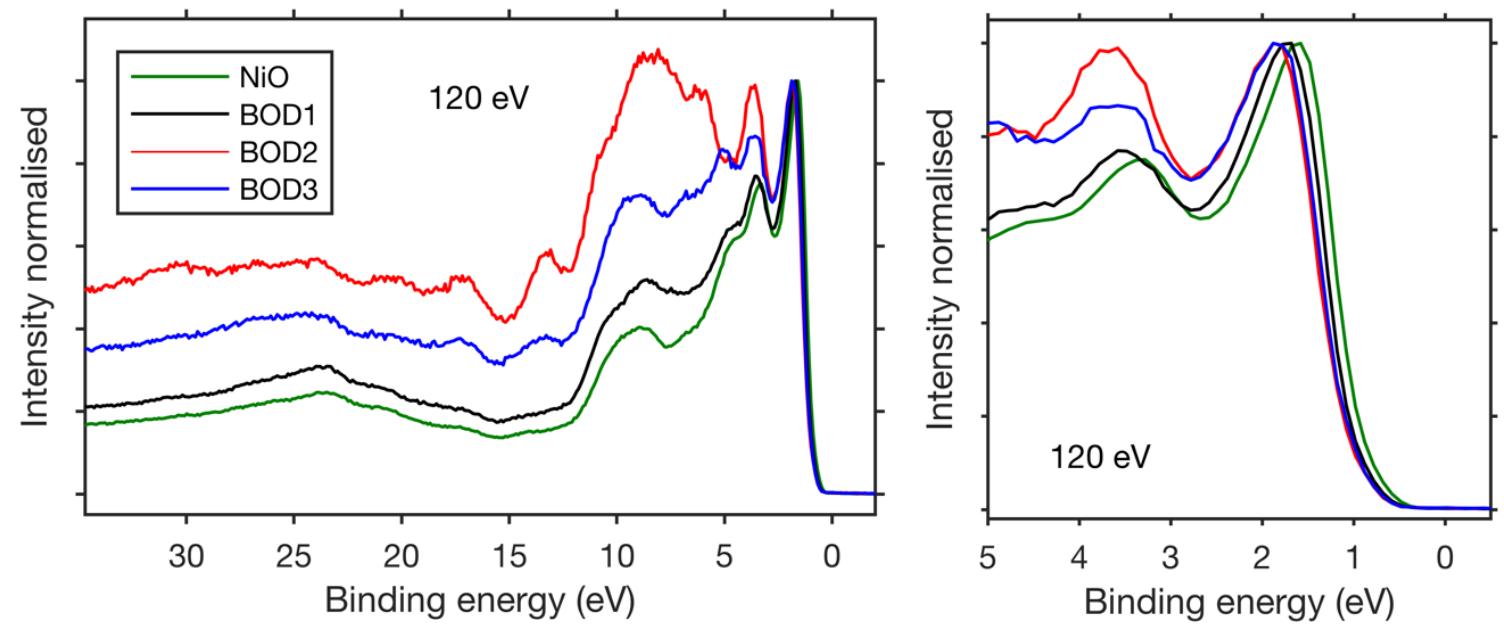

Figure 5. The valence photoelectron spectra for NiO|BOD1, NiO|BOD2, NiO|BOD3 and NiO only measured with a photon energy of $120 \mathrm{eV}$ X-ray excitation energy (left). Expansion of the lower valence region $-0.5-5 \mathrm{eV}$ (right) of the four samples. The spectra were calibrated against an external Au reference and normalised to the height of the lowest binding energy valence band peak.

Figure S22 and 5 show the valence photoelectron spectra for the dye-sensitized $\mathrm{NiO}$ and $\mathrm{NiO}-$ only samples at excitation energies of 2200 and $120 \mathrm{eV}$. As for the dye|FTO samples above, the shape of the spectra varied with excitation energy. With $2200 \mathrm{eV}$ the major contributions are from NiO. ${ }^{26}$ With $120 \mathrm{eV}$, the intensity of the signals associated with the valence orbitals of the dyes increased, but the HOMOs lie underneath the NiO valence band. While the FTO core levels were unaffected by the dye, the $\mathrm{NiO}$ core levels and the valence band edge were shifted to higher binding energy (about $0.1 \mathrm{eV}$ ) when the dyes were absorbed (Table $\mathrm{V}$ and Figure S22 and 5). This means that the dyes do not change the Fermi level on FTO while they slightly shift the Fermi level of $\mathrm{NiO}$ away from the valence band. The shift is consistent with changes at the semiconductor surface on adsorption of the dye, such as displacement of surface hydroxyl residues and the reduction of $\mathrm{Ni}^{3+}$ surface states, which alter the valence band edge energy of the $\mathrm{NiO} \cdot{ }^{27}$ To compare the energy alignment of the dyes on $\mathrm{NiO}$, it is important to consider the core level shifts of both the dyes and the $\mathrm{NiO}$, as the position of the dye levels changes relative to the Fermi level on FTO and on NiO. Figure 6 and Figure S24 
show a comparison of the dye-only valence spectra (determined from FTO, see paragraph above) to the $\mathrm{NiO}$ valence band spectra at $120 \mathrm{eV}$. To enable this comparison, the valence band spectrum of $\mathrm{NiO}$ was shifted to higher binding energies (by $0.1 \mathrm{eV}$, the shift determined from the Ni2p core level), while the dyes were shifted to lower binding energies by the energy difference observed in the $\mathrm{C} 1$ s core level position between FTO and $\mathrm{NiO}$ (Table IV). From this analysis, it can be seen that the HOMO orbitals overlap with the $\mathrm{NiO}$ valence band (Figure 5), and BOD2 shows the deepest HOMO level (i.e. higher binding energy) in agreement with the electrochemistry.

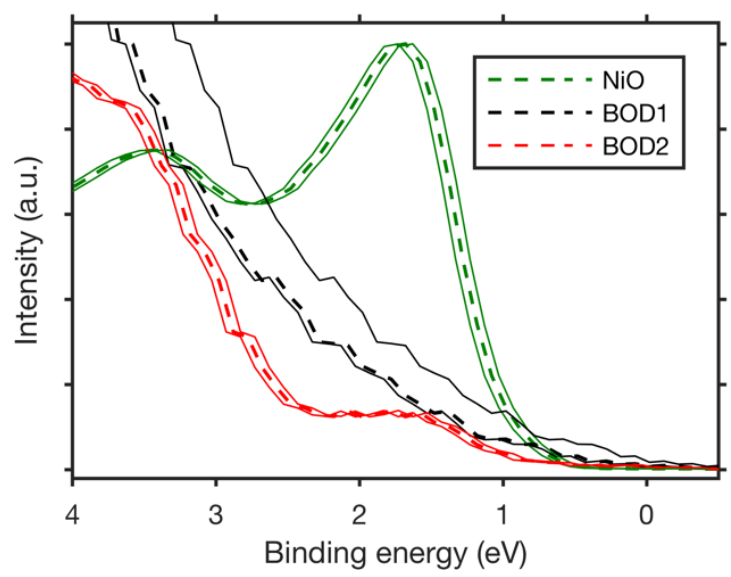

Figure 6. Dye only valence spectra overlaid with $\mathrm{NiO}$ valence band at $120 \mathrm{eV}$. NiO was shifted by $0.1 \mathrm{eV}$, BOD1 by $-0.4 \mathrm{eV}$ and BOD2 by $-0.5 \mathrm{eV}$. Solid lines give the confidence intervals of the shifts. 

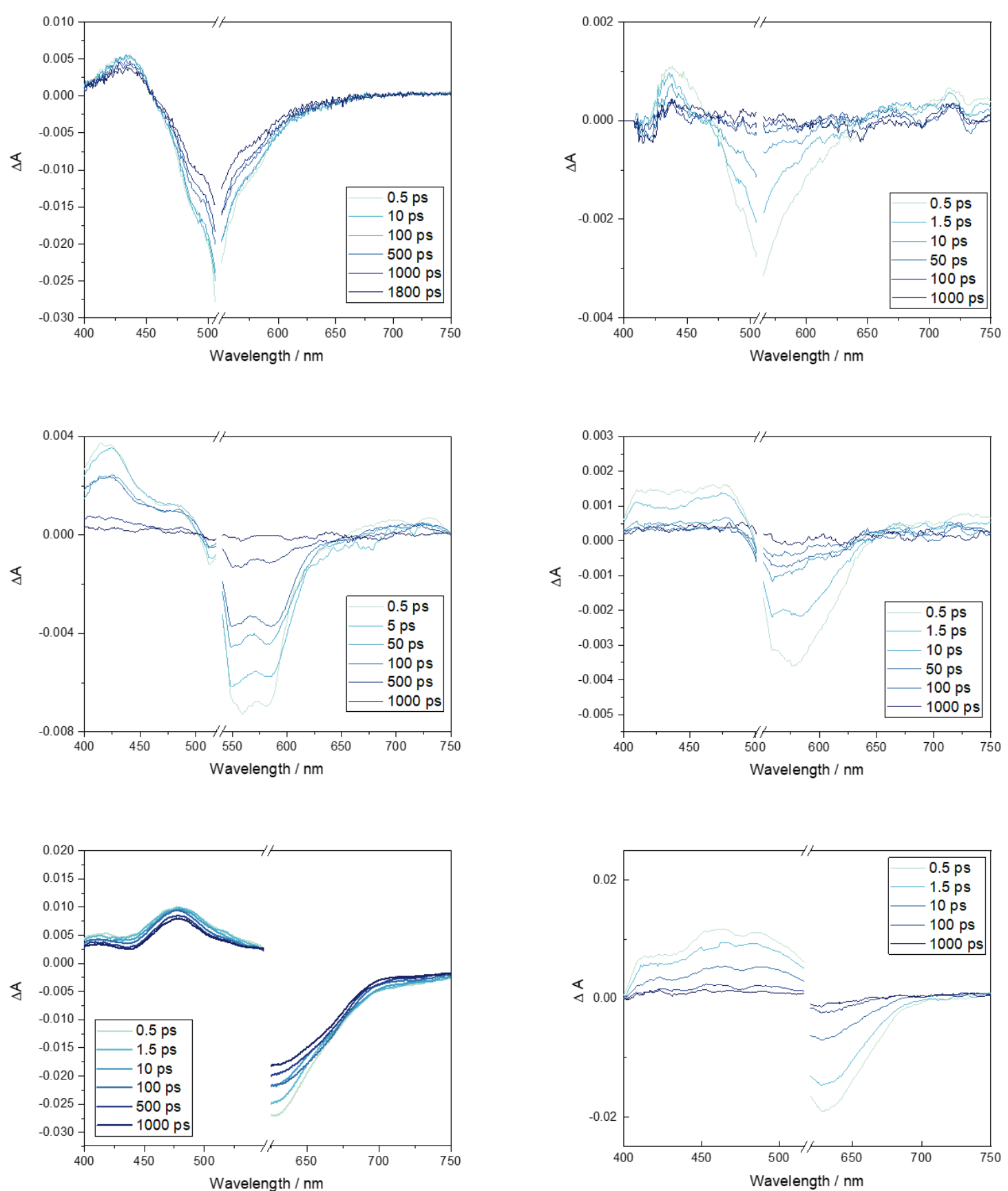

Figure 7. Sub-ps resolution transient absorption spectra of BOD1 (top), BOD2 (middle) and BOD3 (bottom) at selected time delays. Left: in $\mathrm{CH}_{3} \mathrm{CN}$ solution; right: adsorbed onto $\mathrm{NiO}$ film with LiTFSI in acetonitrile electrolyte. (Excitation wavelengths: BOD1 and BOD2 $\lambda_{\mathrm{ex}}=$ $537 \mathrm{~nm}$; BOD3 $\left.\lambda_{\mathrm{ex}}=600 \mathrm{~nm}\right)$. 
To evaluate the rate of photoinduced electron transfer at the dye $\mid \mathrm{NiO}$ interface, sub psresolution transient absorption spectroscopy was performed with solutions of the BOD1-3 in acetonitrile or dye $\mid \mathrm{NiO}$ films, using an excitation wavelength of $\lambda_{\mathrm{ex}}=537 \mathrm{~nm}$ for BOD1 and BOD2 and $\lambda_{\mathrm{ex}}=600 \mathrm{~nm}$ for BOD3. The transient spectra at different delay times after excitation are shown in Figure 7, the decay was fitted to the minimum number of components, the decay associated spectra are provided in Figures S29 - S31, and the lifetimes are summarized in Table VI. The transient spectra for BOD1 and BOD3 contained a characteristic bleach corresponding to the ground state absorption, a negative signal to longer wavelength corresponding to stimulated emission and a positive band to shorter wavelength, ca. 400-470 $\mathrm{nm}$, all of which did not decay within the $1 \mathrm{~ns}$ timescale of the experiment, consistent with the typical excited state lifetime of a bodipy $\left(\mathrm{T} \mathrm{BOD}^{*}=\right.$ ca. $\left.6 \mathrm{~ns}\right) \cdot{ }^{14}$ The transient spectra for BOD2 were similar in shape to those of BOD1 and BOD3 but the bands decayed fully within the 2 ns time resolution of the experiment and the excited state lifetime was $\mathrm{T}=270 \mathrm{ps}$. This is consistent with the weak photoluminescence of BOD2*. The lifetime is much shorter than that of a typical bodipy dye, probably because of the ability of the pendant phenyl substituents to rotate freely, providing non-radiative decay pathways, and the tendency for the dye to aggregate in solution.

The transient spectra of the films, BOD1|NiO, BOD2 $\mid \mathrm{NiO}$ and BOD3|NiO in contact with inert electrolyte of LiTFSI $(0.1 \mathrm{M})$ all decayed within the time resolution of the experiment, indicating a new excited state decay pathway was present. The transient absorption for the dye|NiO films were similar in shape between $400-500 \mathrm{~nm}$ to the reduced dyes generated electrochemically (Figure S26-S28), for BOD1|NiO, e.g. there is a ca. $10 \mathrm{~nm}$ blue shift of the transient absorption maximum compared to BOD1*), and a weak, broad absorption was observed in the red region. This is consistent with the formation of the charge separated state, $\mathrm{BOD} \mid \mathrm{NiO}^{+}{ }^{28}$ The transient absorption decayed fastest for BOD1|NiO followed by BOD2|NiO and BOD3|NiO. The negative signal corresponding to stimulated emission decayed much faster $\left(\mathrm{T}\left(B O D^{*}\right)<2 \mathrm{ps}\right)$. 
This is consistent with rapid electron transfer from NiO to BOD1-3, but the short lifetime of $\mathrm{BOD}-\mid \mathrm{NiO}^{+}$indicates that charge-recombination is also fast.

The addition of a drop of $\mathrm{I}_{3}^{-} / \mathrm{I}^{-}$electrolyte in acetonitrile to the films led to a change in the lifetimes of some of the components. The lifetime of the shortest component $\left(\mathrm{T}_{1}\right)$, which we assigned to the excited state $\mathrm{BOD}^{*}$, was unchanged. For $\mathrm{I}_{3}{ }^{-} / \mathrm{I}^{-}|\mathrm{BOD} 1| \mathrm{NiO},\left(\mathrm{T}_{2}\right)$, which was assigned to the charge-separated state, was still present but a nanosecond component, $\left(\mathrm{T}_{3}>\right.$ $2 \mathrm{~ns})$, appeared and the amplitudes were slightly enhanced compared to the samples with inert electrolyte. $\mathrm{T}_{3}$ contained a ground state bleach, no stimulated emission, and the transient absorption matched the reduced dye, BOD1: The reduced electrolyte species $\mathrm{I}_{2}^{--}$, which should absorb at $425 \mathrm{~nm}$ was not observed on this short timescale. ${ }^{28}$ This suggests that adding the redox electrolyte increases the lifetime of the reduced dye, rather than decreasing it by oxidative quenching. A long lived $\mathrm{T}_{3}$ was also observed for $\mathrm{I}_{3}{ }^{-} / \mathrm{I}^{-}|\mathrm{BOD} 2| \mathrm{NiO}$ and $\mathrm{I}_{3}{ }^{-} / \mathrm{I}^{-}|\mathrm{BOD} 3| \mathrm{NiO}$, and in the latter case the transient spectra lived longer than the resolution of the instrument.

Table VI. Lifetimes for BOD1-3 in acetonitrile or dye|NiO films in contact with both inert and redox electrolyte estimated from global analysis.

\begin{tabular}{|c|c|c|c|}
\hline & $T_{1} / p S$ & $\mathrm{~T}_{2} / \mathrm{ps}$ & $T_{3} / p s$ \\
\hline BOD1 & 0.5 & 50 & inf \\
\hline BOD1|NiO & 2.1 & 248 & \\
\hline $\mathrm{I}_{3}{ }^{-} / \mathrm{I}^{-}|\mathrm{BOD} 1| \mathrm{NiO}$ & 1.3 & 30 & 2790 \\
\hline BOD2 & 1.2 & 270 & \\
\hline BOD2|NiO & 1.1 & 12 & 661 \\
\hline $\mathrm{I}_{3}{ }^{-} / \mathrm{I}^{-}|\mathrm{BOD} 2| \mathrm{NiO}$ & 1.1 & 26 & 3000 \\
\hline BOD3 & 2.2 & 81 & inf \\
\hline BOD3|NiO & 1.9 & 18 & 100 \\
\hline $\mathrm{I}_{3}{ }^{-} / \mathrm{I}^{-} \mid \mathbf{B O D} 3 \mathrm{NiO}$ & 3.4 & 780 & inf \\
\hline
\end{tabular}




\section{Discussion}

The solar cell characteristics were typical for p-type dye-sensitized solar cells, partly due to the low built-in potential of the devices from the small potential difference between the $\mathrm{NiO}$ valence band and the redox potential of the electrolyte. ${ }^{8}$ The $V_{\mathrm{OC}}$ for all three $\mathrm{p}$-DSSCs decreased with increasing $\mathrm{I}_{3}{ }^{-}$concentration, suggesting that charge-recombination at the electrolyte/electrode interface increases. The dark $J$ - $V$ curves are provided in the supporting information, and the increased dark $\mathrm{J}$ for devices with the higher $\mathrm{I}_{3}{ }^{-}$concentration is consistent with this, however it is counterintuitive that increasing the concentration of oxidized species should increase recombination at the photocathode/electrolyte interface. We have previously proposed that the increased recombination could be due to recombination with free iodine in the electrolyte. ${ }^{29}$ The dark current was lowest and the $\mathrm{V}_{\mathrm{OC}}$ was highest for BOD1, which suggests that the alkyl substituents protect the surface of the photocathode from the electrolyte. While it is possible that the valence band may be shifted according to the different dipoles arising from the dye at the surface, the PES data show that the shifts are similar with all the dyes (Figure S25).

A combination of soft and hard X-ray Photoelectron Spectroscopy was applied for the first time to study the frontier orbital alignment or organic photosensitizers with valence states in $\mathrm{NiO}$. Typically, the energy levels in photoelectrochemical devices are estimated from solutionbased experiments. While vacuum experiments have limitations, especially when comparing to photoelectrochemical reactions in solution, we note that the trends in energy (vacuum) are consistent with the trends in electrochemical potential (solution) and solar cell results. Advantages of the technique include the surface sensitivity of the PES and the extra information in electronic environment. By studying the dye adsorbed onto $\mathrm{NiO}$, it was possible to examine the alignment of the HOMO levels relative to the $\mathrm{NiO}$ valence band. The relative HOMO position and dye loading from the PES were consistent with the electrochemical measurements and UV-visible absorption spectroscopy. We observed that adsorption of the dye on the NiO surface has a small but significant effect on the band edge position relative to 
the Fermi level at the dye|semiconductor interface. This shift in the valence band edge could be due to a displacement of surface groups such as $-\mathrm{OH}$, or an electron withdrawing effect induced by the dipole of the dye. The shift in band edge should affect the driving force for electron transfer at the interface, potentially decreasing the $\Delta G_{\mathrm{inj}}$. BOD2 was found to have higher loading on the $\mathrm{NiO}$ surface and we could fully resolve the HOMO for this dye and compare it to $\mathrm{NiO}$. The relative position is consistent with the estimated driving force $\left(\Delta G_{\mathrm{inj}} \approx\right.$ $-1.2 \mathrm{eV}$ ) and the BOD2 $\mathrm{HOMO}$ overlaps with the $\mathrm{NiO}$ valence states.

The photocurrent densities were disappointing given the favorable driving force for charge transfer at the interfaces between the dye and the semiconductor and the dye and the redox mediator. Despite that BOD3 absorbed furthest towards to red region of the visible spectrum $\left(\lambda_{\max }=625 \mathrm{~nm}\right)$, BOD2 produced the highest $J_{\mathrm{Sc}}$ of the three bodipy sensitizers, which was consistent with this dye having the highest absorbance and the highest driving force for charge separation. BOD2 had the lowest absorption coefficient in solution of the three bodipy dyes but the highest $L H E$, in agreement with the highest loading on the NiO surface within this series, as shown by PES. BOD1 produced the lowest $J_{\text {SC }}$ and the lowest $L H E$. Increasing the concentration of $\mathrm{I}_{2}$ added to the electrolyte to $0.5 \mathrm{M}$ lead to an increase in the $J_{\mathrm{sc}}$ for all three bodipy sensitizers and the trend in $J_{S C}$ followed that of the LHE (BOD2 > BOD3 > BOD1). This suggests that the $J_{S c}$ is limited by the dye-regeneration step, i.e. the interception of the electron in the photoreduced dye by $\mathrm{I}_{3}{ }^{-}$in the electrolyte, when the $\mathrm{I}_{3}{ }^{-}$concentration was $\sim 0.1$ M. When the concentration of $\mathrm{I}_{3}^{-}$was increased to $\sim 0.5 \mathrm{M}$, the current was limited by the amount of dye on the surface. Transient absorption spectroscopy was used to investigate the electron transfer dynamics to rationalize this.

The transient absorption spectra show that light-induced charge-separation at the dye $\mid \mathrm{NiO}$ interface is rapid $\left(<5 \mathrm{ps}\right.$ ). Charge-separation forming $\mathrm{BOD}^{-} \mid \mathrm{NiO}^{+}$is more than an order of magnitude faster than the excited state lifetime for the BOD1-3, suggesting that this process is efficient in all three systems. This is consistent with our electrochemistry and PES experiments, which show that electron transfer from the valence band of $\mathrm{NiO}$ to the lowest 
partially filled orbital of the excited dye is thermodynamically favorable for all three dyes. In the absence of redox electrolyte, the recombination of the charge separated state is also very fast, and the reduced dye is immediately quenched. This is consistent with the low photocurrent density recorded for the BOD1-3-sensitized solar cells. The kinetics are heterogeneous, which is typical for dye-sensitized mesoscopic systems. The rapid recombination for dye-sensitized $\mathrm{NiO}$, which is in contrast to much longer-lived charge separation in dye-sensitized $\mathrm{TiO}_{2}$ systems, is believed to be caused by recombination to an intra-bandgap state. ${ }^{30}$ Liu et al. proposed that $\Delta G_{\mathrm{inj}} \sim-0.8 \mathrm{eV}$ is required for high chargeseparation yields. ${ }^{31}$ Despite the substantial $\Delta G_{\mathrm{inj}}$ for BOD2 $(-1.2 \mathrm{eV})$, the lifetime of the excited state is shortest for this dye, suggesting that injection is not in the Marcus inverted region. Possibly this large overpotential leads to injection into deeper lying states in the NiO. Chargerecombination with this state would lie in the Marcus inverted region and, therefore, be slower than recombination with the intra-bandgap state..$^{30}$

Another reason for the rapid recombination could be the electronic structure of the excited state of BOD1-3. The DFT calculations reveal the spread of the LUMO across the anchoring group and the chromophore, which could promote electron transfer from NiO back to the dye. This electronic configuration would be ideal for $\mathrm{TiO}_{2}$, but a better configuration for $\mathrm{p}$-type systems, in which the direction of electron transfer is reversed, could be to have the electron density located on the anchoring group in the HOMO and not in the LUMO. This can be readily achieved by placing the anchoring group on the pyrrole substituents. To improve chargeseparation, an electron withdrawing substituent could be placed at the meso position to pull electron density away from the $\mathrm{NiO}$ surface.

The lifetime of the BOD $\mid \mathrm{NiO}^{+}$increased slightly in the presence of the redox electrolyte, rather than decreased as would be expected when the electron in the dye would be intercepted by the $\mathrm{I}_{3}$. Previous work by Gibson et al. showed that $\mathrm{I}^{-}$is oxidized at the surface of $\mathrm{NiO}$, resulting in the immediate formation of triiodide even without an applied bias, which suggests that $\mathrm{Ni}^{3+}$ states at the surface are reduced. ${ }^{29}$ Tian et al. demonstrated that $\mathrm{Ni}^{3+}$ surface states of $\mathrm{NiO}$ 
can be reduced by other chemical reductants such as sodium borohydride, and that $E\left(\mathrm{Ni}^{3+} / \mathrm{Ni}^{2+}\right)$ lies ca. $200 \mathrm{mV}$ more positive than $E\left(\mathrm{I}_{3}{ }^{-} / \mathrm{l}^{-}\right) .{ }^{27}$ We suggest here that reducing the $\mathrm{Ni}^{3+}$ surface states blocks the fast recombination pathway and leads to a longer BOD-|NiO' lifetime. This behavior was previously shown for a cationic dye, but here we show that this observation also applies to neutral dyes. ${ }^{32}$ This is consistent with processes occurring at the electrolyte|electrode interface rather than electrolyte|dye interface. Figure 8. illustrates these competing recombination pathways for dyes adsorbed onto $\mathrm{NiO}$ with and without electrolyte present. Nonetheless, even in the presence of electrolyte, the amplitude of the signal in the ns timescale is small compared to that immediately following excitation. This suggests that only a small amount of reduced dye survives long enough to react with the electrolyte, leading to low $J_{S c}$ in the devices.

a)

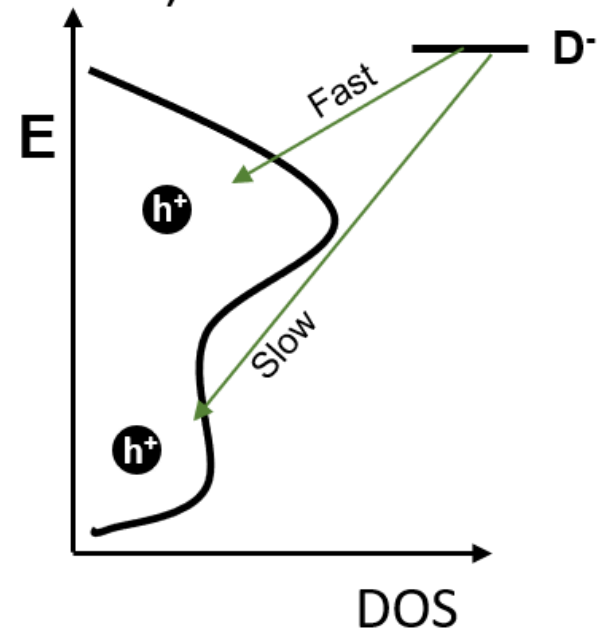

b)

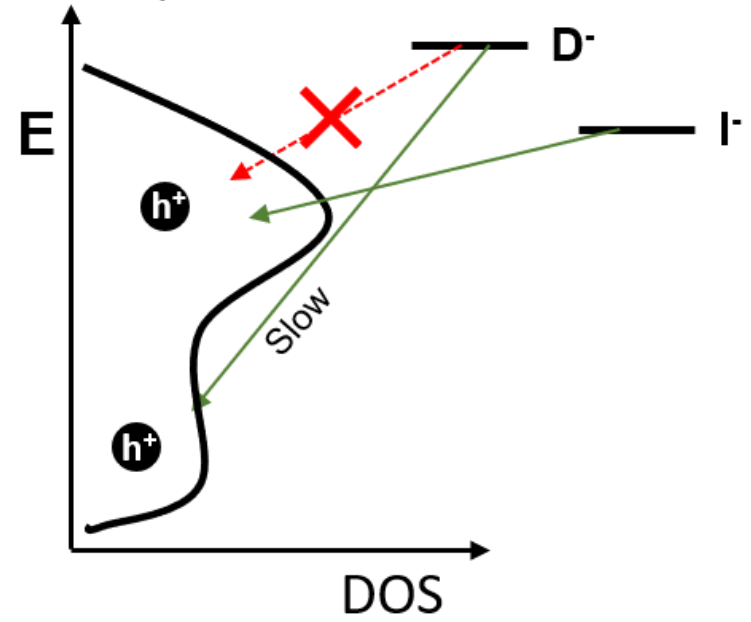

Figure 8. Scheme proposing two competing charge recombination processes at the interface between the dye and $\mathrm{NiO}$ in a p-DSSC; a) without iodine electrolyte; b) with iodine electrolyte. In (b), reduction of surface $\mathrm{Ni}^{3+}$ leads to slower recombination, increasing the lifetime of the reduced dye. 


\section{Conclusions}

This paper outlines a surface sensitive approach to investigate the behavior at the interfaces in p-type dye sensitized solar cells. A series of bodipy dyes, BOD1-3, with different structural and electronic properties, each able to anchor onto the surface of $\mathrm{NiO}$ were studied both in solution and on surfaces. All three dyes displayed favorable properties for use in p-DSSCs, such as reversible electrochemistry in solution, high extinction coefficients and suitably placed frontier orbitals to promote electron injection from the $\mathrm{NiO}$ and regeneration by the triiodide/iodide electrolyte. The $J_{\mathrm{sc}}$ values appeared to be limited by the dye regeneration in a system with low concentrations of $\mathrm{I}_{3}{ }^{-}$in the electrolyte. BOD2 was least affected by the reduction of iodine in the electrolyte composition indicating that the regeneration of BOD2 in the p-DSSC was most efficient out of the three dyes. Additionally, according to the PES data, driving force for charge separation was highest for BOD2, which agrees with the solar cell results.

We compared the trends in driving force for electron transfer at the dye $\mid \mathrm{NiO}$ and dyelelectrolyte interfaces with the lifetimes of the charge transfer and used the trends in structural and electronic properties to rationalize the solar cell characteristics. The initial charge separation process was fast $(<5 \mathrm{ps})$, however in absence of the redox electrolyte the recombination of charges in the dye and semiconductor is also very fast $(<2 \mathrm{~ns})$. In the presence of electrolyte, the lifetime for the charge-separated state increased into the ns timescale for all three dyes. We propose that the electrolyte passivates the surface states responsible for the fast recombination pathway.

We hope that the approach and results in this manuscript provide guidelines on how to effectively study the interfaces found in these devices. As the trend in research into p-DSSC moves towards looking for new p-type semiconducting materials to increase the performance of the devices, systematic studies such as this, especially involving photoelectron spectroscopy with soft X-rays to probe the interface, should enable us to understand more about photosensitization of $p$-type semiconductors, which is much less understood compared 
to n-type materials such as $\mathrm{TiO}_{2}, \mathrm{ZnO}$ and $\mathrm{SnO}_{2}$. Meanwhile, engineering of the photosensitizer to overcome the challenges of $\mathrm{NiO}$ is necessary if alternative $\mathrm{p}$-type metal oxides with more favorable properties cannot be found.

\section{Supplementary Information}

Experimental details, NMR, UV-visible absorption and emission spectra, cyclic voltammetry, spectroelectrochemistry, PES data, DSSC $J-V$ curves and TAS.

\section{Author contributions}

NP synthesised the molecules, performed the optical and electrochemical studies, assembled and tested the solar cells, interpreted the TAS and PES data and co-wrote the manuscript. VDA performed the DFT calculations. EAG designed the study and co-wrote the manuscript. BD directed the TAS experiments. MW and RAW performed the TAS experiments and fitted the data. UBC designed the PES experiments. TS performed the PES experiments.

\section{Acknowledgments}

EAG and NP thank Newcastle University for a PhD studentship, The North East Centre for Energy Materials EP/R021503/1, ERC starting grant, p-TYPE 715354, and STFC. U.B.C. and T.S. acknowledge financial support from the Swedish Research Council (VR 2018-04125) and the Göran Gustafsson foundation. This work was carried out with the support of the Diamond Light Source, instrument I09 (proposals SI18517 and SI22807). We also thank Pradeep T. Kumar and Tien-Lin Lee for support at the beamline. The research leading to this result has been supported by the project CALIPSOplus under Grant Agreement 730872 from the EU Framework Programme for Research and Innovation HORIZON 2020. 


\section{Data availability}

The data that support the findings of this study are openly available in data.ncl.ac.uk at http://doi.org/10.25405/data.ncl.c.5073989.

\section{References}

${ }^{1}$ A. Hagfeldt, G. Boschloo, L. Sun, L. Kloo, and H. Pettersson, Chem. Rev. 110, 6595 (2010).

${ }^{2}$ G. Hashmi, K. Miettunen, T. Peltola, J. Halme, I. Asghar, K. Aitola, M. Toivola, and P. Lund, Renew. Sustain. Energy Rev. 15, 3717 (2011).

${ }^{3}$ E. Biyik, M. Araz, A. Hepbasli, M. Shahrestani, R. Yao, L. Shao, E. Essah, A.C. Oliveira, T. del Caño, E. Rico, J.L. Lechón, L. Andrade, A. Mendes, and Y.B. Atlı, Eng. Sci. Technol. an Int. J. 20, 833 (2017).

${ }^{4}$ M. Pagliaro, R. Ciriminna, and G. Palmisano, ChemSusChem 1, 880 (2008).

${ }^{5}$ M. Freitag, J. Teuscher, Y. Saygili, X. Zhang, F. Giordano, P. Liska, J. Hua, S.M. Zakeeruddin, J.-E. Moser, M. Grätzel, and A. Hagfeldt, Nat. Photonics 11, 372 (2017).

${ }^{6}$ K. Kakiage, Y. Aoyama, T. Yano, K. Oya, J. Fujisawa, and M. Hanaya, Chem. Commun. 51, 15894 (2015).

7 J. He, H. Lindström, A. Hagfeldt, and S.-E. Lindquist, Sol. Energy Mater. Sol. Cells 62, 265 (2000).

${ }^{8}$ E. Benazzi, J. Mallows, G.H. Summers, F.A. Black, and E.A. Gibson, J. Mater. Chem. C 7, 10409 (2019).

${ }^{9}$ L. Li, E.A. Gibson, P. Qin, G. Boschloo, M. Gorlov, A. Hagfeldt, and L. Sun, Adv. Mater. 22, 1759 (2010).

${ }^{10}$ M. Wykes, F. Odobel, C. Adamo, I. Ciofini, and F. Labat, J. Mol. Model. 22, (2016). 
${ }^{11}$ Y. Farré, M. Raissi, A. Fihey, Y. Pellegrin, E. Blart, D. Jacquemin, F. Odobel, Y. Farre, M. Raissi, A. Fihey, Y. Pellegrin, E. Blart, D. Jacquemin, Y. Farré, M. Raissi, A. Fihey, Y. Pellegrin, E. Blart, D. Jacquemin, F. Odobel, Y. Farre, M. Raissi, A. Fihey, Y. Pellegrin, E. Blart, D. Jacquemin, Y. Farré, M. Raissi, A. Fihey, Y. Pellegrin, E. Blart, D. Jacquemin, and F. Odobel, ChemSusChem 10, 2618 (2017).

${ }^{12}$ C.J. Wood, M. Cheng, C.A. Clark, R. Horvath, I.P. Clark, M.L. Hamilton, M. Towrie, M.W. George, L. Sun, X. Yang, and E.A. Gibson, J. Phys. Chem. C 118, 16536 (2014).

${ }^{13}$ C.J. Wood, G.H. Summers, and E.A. Gibson, Chem. Commun. 51, 3915 (2015).

${ }^{14}$ G.H. Summers, J.-F. Lefebvre, F.A. Black, E. Stephen Davies, E.A. Gibson, T. Pullerits, C.J. Wood, and K. Zidek, Phys. Chem. Chem. Phys. 18, 1059 (2016).

${ }^{15}$ H. Kim, A. Burghart, M.B. Welch, J. Reibenspies, and K. Burgess, Chem. Commun. 1889 (1999).

${ }^{16}$ Y. Kubo, Y. Minowa, T. Shoda, and K. Takeshita, Tetrahedron Lett. 51, 1600 (2010).

17 Y. Kubo, K. Watanabe, R. Nishiyabu, R. Hata, A. Murakami, T. Shoda, and H. Ota, Org. Lett. 13, 4574 (2011).

${ }^{18}$ Y. Tomimori, T. Okujima, T. Yano, S. Mori, N. Ono, H. Yamada, and H. Uno, Tetrahedron 67, 3187 (2011).

${ }^{19}$ Z. Feng, L. Jiao, Y. Feng, C. Yu, N. Chen, Y. Wei, X. Mu, and E. Hao, J. Org. Chem. 81, 6281 (2016).

${ }^{20}$ S. Saino, M. Saikawa, T. Nakamura, M. Yamamura, and T. Nabeshima, Tetrahedron Lett. 57,1629 (2016).

${ }^{21}$ S. Choi, J. Bouffard, and Y. Kim, Chem. Sci. 5, 751 (2014).

${ }^{22}$ E.A. Gibson, L. Le Pleux, J. Fortage, Y. Pellegrin, E. Blart, F. Odobel, A. Hagfeldt, and G. Boschloo, Langmuir 28, 6485 (2012). 
M. Hahlin, E.M.J. Johansson, S. Plogmaker, M. Odelius, D.P. Hagberg, L. Sun, H. Siegbahn, and H. Rensmo, Phys. Chem. Chem. Phys. 12, 1507 (2010).

${ }^{24}$ M. Weston, T.J. Reade, K. Handrup, N.R. Champness, and J.N. O’Shea, J. Phys. Chem. C 116, $18184(2012)$

${ }^{25}$ U.B. Cappel, P. Liu, F.O.L. Johansson, B. Philippe, E. Giangrisostomi, R. Ovsyannikov, A. Lindblad, L. Kloo, J.M. Gardner, and H. Rensmo, ChemPhysChem 19, 1041 (2018).

${ }^{26}$ A.N. Mansour, Surf. Sci. Spectra 3, 231 (1994).

${ }^{27}$ L. D’Amario, R. Jiang, U.B. Cappel, E.A. Gibson, G. Boschloo, H. Rensmo, L. Sun, L. Hammarström, and H. Tian, ACS Appl. Mater. Interfaces 9, 33470 (2017).

${ }^{28}$ J.-F. Lefebvre, X.-Z. Sun, J.A. Calladine, M.W. George, and E.A. Gibson, Chem. Commun. 50,5258 (2014).

${ }^{29}$ C.J. Wood, C.A. McGregor, and E.A. Gibson, ChemElectroChem 11, 1827 (2016).

${ }^{30}$ A.L. Smeigh, L. Le Pleux, J. Fortage, Y. Pellegrin, E. Blart, F. Odobel, and L. Hammarström, Chem. Commun. 48, 678 (2012).

${ }^{31}$ Z. Liu, D. Xiong, X. Xu, Q. Arooj, H. Wang, L. Yin, W. Li, H. Wu, Z. Zhao, W. Chen, M. Wang, F. Wang, Y.-B. Cheng, and H. He, ACS Appl. Mater. Interfaces 6, 3448 (2014).

${ }^{32}$ F.A. Black, C.J. Wood, S. Ngwerume, G.H. Summers, I.P. Clark, M. Towrie, J.E. Camp, and E.A. Gibson, Faraday Discuss. 198, 449 (2017). 